\title{
Simulation Technique of Synchronous Reluctance Bearingless Machine
}

\author{
Yulia Domracheva, Sergey Loginov \\ Pskov State University, Electrical Engineering Faculty, Department of Drive and \\ automation systems. \\ Address: Lenin Square 2, Pskov, 180000, Russian Federation
}

\begin{abstract}
Magnetically levitated rotor electric machines can be used under such conditions wherein application of conventional bearings is impossible because of presence of lubrication. Active magnetic bearings application requires rotor length increase and stalling speed is decreased. Bearingless electric machines are the next step up of the magnetically levitated rotor machines. A bearingless electric machine module generates electromagnetic torque and radial forces. This feature makes possible to decrease the rotor length on retention of capacity of torque and forces. It is necessary to verify a control system operation algorithm via simulation model owing to complexity of machine electromagnetic interaction. It is practical to use a variable attribute set model for this purpose. The following article considers one of these models for researching the system via MATLAB Simulink. Basic relations for programmatic model creation are presented. Comparative assessment of various machine model types is made.
\end{abstract}

Keywords - computer simulation, bearingless electric machine, simulation technique, programmatic model.

\section{INTRODUCTION}

Magnetically levitated rotor electric machines (EM) can be used under such conditions wherein application of conventional bearings is impossible because of lubrication presence. On these machines active magnetic bearings (AMB) are currently used. Application $\mathrm{AMB}$ requires increase of the rotor length, thus reducing stalling speed [1].

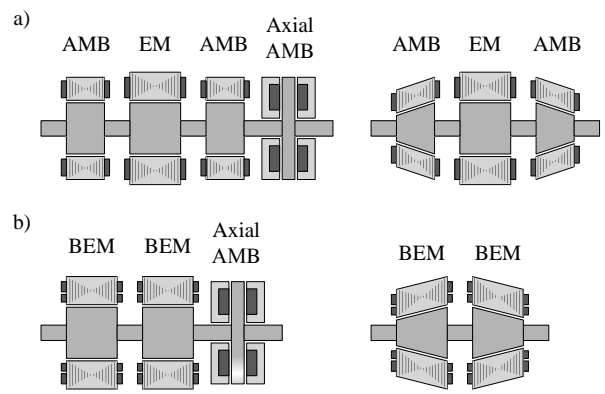

Fig. 1. Magnetically levitated rotor electric machine modifications: a) active magnetic bearing construction variants, b) bearingless electric machines.

Bearingless electric machines (BEM) are the next step up of development the magnetically levitated rotor machines. Into the stator slots of the bearingless module two sets of windings are placed: motor and suspension windings. It enables to generate electromagnetic torque and radial forces, which suspend the rotor at center of the stator. This feature makes it possible to decrease the rotor length on retention of capacity of torque and forces [2]. Overall dimensions decrease is associated with application the field of the motor winding as an integral part of the field of the suspension winding. It also allows to reduce power consumption.
Interference of the radial forces and the torque is an essential disadvantage of BEM. A special construction of the BEM modules makes it possible to control them separately. BEM construction principle was proposed in 1974 [3]. By this definition, one of the stator windings must have $p$ pair of poles, and the other $p+1$. The number of the rotor poles must be equal to the number of the motor winding poles. In this case total electromotive force (EMF), induced by one of the windings in the other winding, is zero. The magnetomotive force (MMF) of the suspension winding doesn't produce a torque. But this mechanism works in case of the central position of the rotor. The MMF distribution becomes unsymmetrical because of displacement of the rotor. As a consequence, the suspension winding field generates a torque and the motor winding field generates a radial force.

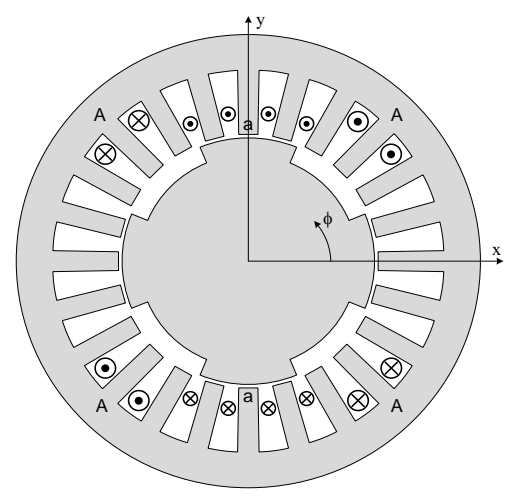

Fig. 2. Cross sectional drawing of synchronous reluctance bearingless machine. The example of winding sets phase placement: a - suspend winding phase, $\mathrm{A}$ - motor winding phase.

Interference of the radial forces and the torque can be reduced by employing a special control algorithm of the winding current. Some versions of 
building such algorithm for a synchronous reluctance bearingless machine (SRBM) were proposed. One of them uses a special compensator, calculating the motor current control signal subject to the suspension current magnitude, and conversely [4]. In 2010 a control system structure had constructed on the basis of an inverse system [5].

SRBM design parameters in all of the proposed control systems has presented with inductances $L_{d}$ and $L_{q}$. Authors of [6] suggest to define relationship between these inductances and displacements $x$ and $y$ by experiment. It allows to obtain sufficiently accurate model to create the control system. There is no feasibility to create design procedure because of lack the analytic formula of inductance in relation to the BEM design parameters.

For creation of the SRBM mathematical model commonly used next conventions: a) reciprocal value of the air gap defined by approximate expression, b) the air gap permeance between rotor poles is zero, c) the MMF upper harmonics and tooth kinks are disregarded, d) the core steel saturation is neglected. Desired precision of the mathematical model creation defined by functionality of the control system, developed on its basis.

Testing control system functionality shouldn't be carried out with model which contains same assumptions. It is easy to use a model with variable array of the attributes for this purpose. At this article one modification of such models for researching the system via MATLAB Simulink is presented. All computations are executed via $\mathrm{C}++$ programming language. Thereby hereinafter it is named programmatic model.

\section{MAIN MODEL EXPRESSIONS}

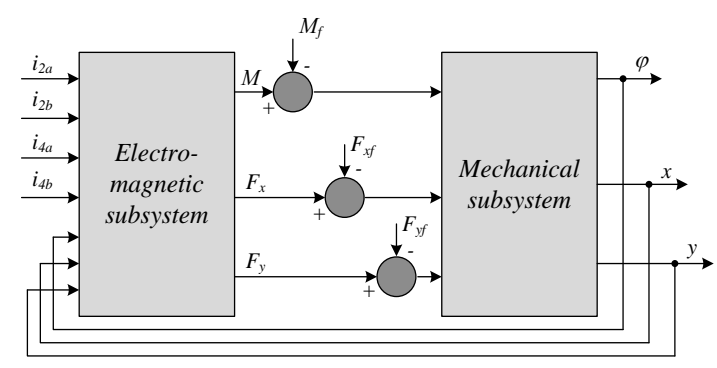

Fig. 3. Variable attribute set model block diagram.

The SRBM model can be divided into two subsystems: electromagnetic and mechanical (fig. 3). The electromagnetic subsystem computes a torque $M$ and radial forces $F_{x}$ and $F_{y}$ in terms of the rotor position and currents of the stator windings. The torque and the forces are added to disturbances, and then arrived at the mechanical subsystem. Output coordinates of the model are a turning angle $\varphi$ and radial rotor displacements $x$ and $y$.

The torque and the radial forces must be computed considering geometry of the windings and the cores. These variables are the partial derivatives of magnetic energy. Magnetic energy equation must consider its dependence on the turning angle and the radial rotor displacements. For the analytical derivation of this expression are used the simplified expression for the air gap reciprocal value. However, in the model must be capability of computation of the air gap value by all means. Therefore the electromagnetic torque and the radial forces are defined as a resultant of torques and forces, applied to some elementary parts of the rotor surface.

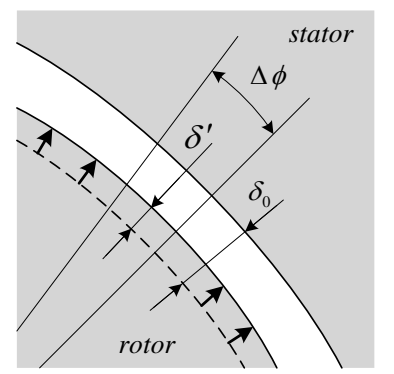

Fig. 4. The SRBM air gap piece. The rotor was displaced on $\delta$. Until this moment the air gap value was equal to $\delta_{0}$.

Let's divide on elements the surface of the rotor. The length of each element is equal to the core reactive part length, and width is equal to the arc length $\Delta \phi$ (fig. 4). The angle must be so small that the air gap value inside of any of the elements may be considered constant. The air gap value depending on the angular coordinate $\phi$ and the radial rotor displacements $x$ and $y$ is defined by next expression.

$$
\delta=\delta_{0}-x \cdot \cos \phi-y \cdot \sin \phi=\delta_{0}-\delta^{\prime}
$$

Where $\delta_{0}$ is the air gap value, when displacements of the rotor are zero. Value of $\delta^{\prime}$ is constant inside of the element $\Delta \phi$. The electromagnetic torque and the radial forces are computed numerically by next expressions.

$$
\begin{aligned}
& M_{i}=\frac{\Delta W_{i}}{\Delta \phi} \\
& F_{x i}=\frac{\Delta W_{i}}{\Delta x} F_{y i}=\frac{\Delta W_{i}}{\Delta y}
\end{aligned}
$$

Where $M_{i}$ is electromagnetic torque, affecting on the element $\Delta \phi_{i} ; F_{x i}$ and $F_{y i}$ are radial forces, affecting on the element $\Delta \phi_{i} ; \Delta W_{i}$ is magnetic energy, stored in the air gap, close to the element $\Delta \phi_{i}$.

$$
\Delta W_{i}=\frac{1}{2} \sum_{j=1}^{N} \sum_{k=1}^{N} \Delta L_{i j k} \cdot i_{j} \cdot i_{k}
$$


Where $N$ is the number of the stator windings; $i$ is a winding current; $\Delta L_{i}$ is a self inductance or mutual inductance of a winding, being defined by the air gap element $\Delta \phi_{i}$.

$$
\Delta L_{i j k}=\frac{\mu_{0} \cdot \Delta \phi_{i} \cdot r \cdot l}{\delta_{0}-\delta^{\prime}} \cdot w_{j} \cdot w_{k}
$$

Where $\mu_{0}=4 \pi \cdot 10^{-7}$ is vacuum permeance; $S=r$ $\cdot \Delta \phi \cdot l$ is an element area; $r$ is the rotor radius; $l$ is the core reactive part length; $w$ is an appropriate winding turns number.

The total electromagnetic torque and radial forces are defined by next equations.

$$
\begin{gathered}
M=\sum_{i=1}^{n} M_{i} \\
F_{x}=\sum_{i=1}^{n} F_{x i} \quad F_{y}=\sum_{i=1}^{n} F_{y i}
\end{gathered}
$$

Where $n$ is the count of the elements.

Computations of the output variables of the electromagnetic subsystem by (1) - (7) are executed every modeling iteration.

The turning angle $\varphi$ and the radial displacements $x$ and $y$ of the rotor at the mechanical subsystem block can be defined by motion equations.

$$
\begin{aligned}
& m \cdot \ddot{x}=F_{x}-F_{x f} \\
& m \cdot \ddot{y}=F_{y}-F_{y f} \\
& J \cdot \ddot{\varphi}=M-M_{f}
\end{aligned}
$$

Where $m$ is the rotor mass; $J$ is the rotor moment of inertia; $F_{x f}, F_{y f}$ and $M_{f}$ are disturbances. Let's obtain equations for computation of the displacements and the turning angle in the step $j$ from (8).

$$
\begin{aligned}
& x_{j}=\frac{T_{k}}{m}\left(F_{x}-F_{x f}\right)+2 x_{j-1}-x_{j-2} \\
& y_{j}=\frac{T_{k}}{m}\left(F_{y}-F_{y f}\right)+2 y_{j-1}-y_{j-2} \\
& \varphi_{j}=\frac{T_{k}}{J}\left(M-M_{f}\right)+2 \varphi_{j-1}-\varphi_{j-2}
\end{aligned}
$$

Where $T_{k}$ is the computation step size.

To improve accuracy of the output model variables computation it is necessary to minimize the step size and the angle $\Delta \phi$.

Via programmatic model it is possible to regard or disregard any attribute of the machine. It enables to investigate its influence on the system performance. Let's consider some ways of research of influence assumptions.

\section{Approximate expression for the air gap reciprocal value}

It has to use an approximate expression for reciprocal value of the air gap to obtain analytic dependences of the inductances from the radial displacements of the rotor. It is because of inconvenience of using the exact equation for the control system creation.

Exact expression for the air gap reciprocal value is presented here.

$$
\frac{1}{\delta}=\frac{1}{\delta_{0}-x \cdot \cos \phi-y \cdot \sin \phi}=\frac{1}{\delta_{0}-\delta^{\prime}}
$$

The approximate expression is obtained by its decomposition for geometric sequence.

$\frac{1}{\delta}=\frac{1}{\left(7 \delta_{0}-\delta^{\prime}\right.}=\frac{1}{\delta_{0}} \cdot \frac{1}{1-\bar{\delta}}=\frac{1}{\delta_{0}} \cdot\left(1+\bar{\delta}+\bar{\delta}^{2}+\ldots\right)$

Where $\bar{\delta}=\delta^{\prime} / \delta_{0}<1$.

Generally in (11) only the first-order summand are used [6]. In this case the air gap value is computed correct only when the rotor displacements are small $\left(\approx 20 \%\right.$ from $\left.\delta_{0}\right)$. There are essential inaccuracy in other cases. By using the second-order summand in (11) the air gap value can be exactly computed when the displacements are equal to $30 \%$ from $\delta_{0}$. The using of approximate expression for the (8ir gap value in the model should to exclude influence of computation accuracy on work of the control system, created according to this expression. It may be carried out by recounting $\delta^{\prime}$. Having assimilated the exact and approximate expressions for the air gap value, we obtain next relation.

$$
\delta^{\prime}=\delta_{0} \cdot \frac{\bar{\delta}}{1+\bar{\delta}}
$$

By doing likewise, we obtain relation, when using the second-order summand in (11).

(9)

$$
\delta^{\prime}=\delta_{0} \cdot \frac{\bar{\delta}+\bar{\delta}^{2}}{1+\bar{\delta}+\bar{\delta}^{2}}
$$

\section{A. $\quad$ The air gap permeance between the} rotor poles

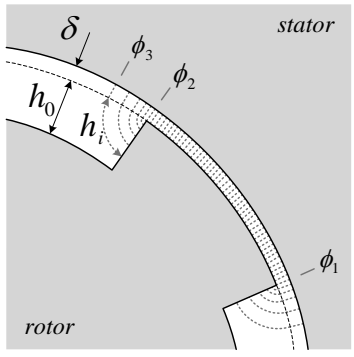

Fig. 5. The air gap piece of SRBM. Main magnetic flux comes through the poles surface, because of smallness of the air gap. 
In the SBRM mathematical model, used for the control system creation, the air gap permeance between the rotor poles is neglected. In this case, equations (6) and (7) include summands which describe exposure to the elements, located on the pole surface: from $\phi_{1}$ to $\phi_{2}$ (fig. 5).

It has to include corresponding summands in equations (6) and (7) to considered the air gap permeance between the poles. For example, the elements from $\phi_{2}$ to $\phi_{3}$ must be included in these equations to consider the permeance of the lateral pole surface. In this case, the air gap value is increased by the value $h_{i}$, which can be defined according to a path shape of the flux [7]. In the range of the element $\Delta \phi_{i}$ the value $h_{i}$ is constant. Therefore the equations model are not changed. But, should be taken into account next modification.

$$
\delta=\delta_{0}^{\prime}-\delta^{\prime}=\delta_{0}+h_{i}-\delta^{\prime}
$$

\section{MMF upper harmonics and tooth kinks}

The output parameters of the electromagnetic subsystem are computed numerically; therefore the MMF shape changes don't require modification of the computing algorithm. Upper harmonics are added to the first harmonic in the equation magnetic energy as optional summands.

Tooth kinks can be considered by adding the MMF harmonic, which have order equal to the stator slots number. If the stator slots are allocated uneven or there is an essential difference between tooth and slot width, tooth kinks should be modeled by changing the air gap permeance.

\section{Core steel saturation}

The iron core reluctance must be considered by adding it consecutively to the air gap reluctance. In the proposed model conveniently bring it to the air gap value.

$$
R_{\delta}+R_{c}=\frac{\delta_{0}-\delta^{\prime}}{\mu_{0} \cdot S}+R_{c}=\frac{\delta_{0}-\delta^{\prime}+\mu_{0} \cdot S \cdot R_{c}}{\mu_{0} \cdot S}
$$

Where $R_{\delta}$ is the air gap reluctance; $R_{c}$ is the iron cores reluctance. Therefore the air gap value is described by next expression.

$$
\delta=\delta_{0}-\delta^{\prime}+\mu_{0} \cdot S \cdot R_{c}
$$

The core steel saturation can be considered by changing the iron core reluctance depending on an applied MMF.

$$
F_{c j}=F_{\Sigma} \cdot \frac{\mu_{0} \cdot S \cdot R_{c j-1}}{\delta_{0}-\delta^{\prime}+\mu_{0} \cdot S \cdot R_{c j-1}}
$$

Where $F_{c j}$ is the drop in MMF in the cores of the stator and rotor on $j$ iteration; $F_{\Sigma}$ is total MMF of the windings; $R_{c j-1}$ is iron core reluctance on $j-1$ iteration.

(A4relation $R_{c}=f\left(F_{c}\right)$ is created according to the characteristic of the magnetization iron. When computation the model via program, it's easy to prescribe this relation both numerically and by an approximate expression. After computation $F_{c j}$ is defined new value of $R_{c j}$.

$$
R_{c j}=f\left(F_{c j}\right)
$$

Computation $F_{c j}$ with (17) by (17) introduces an error in the steel reluctance compute owing to use $R_{c}$ value, obtained on previous iteration. Therefore, if the MMF changes drastically, it has to reiterate the computations with (17) and (18) several times for accuracy increase.

\section{CONCLUSION}

The research of electromechanical system can be carried out via models various types: an analytical model, a finite element method (FEM) model, a programmatic model. Every one of them has benefits and drawbacks. Let's compare them in terms

\begin{tabular}{|c|c|c|c|}
\hline $\begin{array}{ll}\text { Assumption } & \text { Model } \\
\end{array}$ & Analytical model & $\begin{array}{l}\text { Finite element method } \\
\text { model }\end{array}$ & $\begin{array}{l}\text { Programmatic } \\
\text { model }\end{array}$ \\
\hline $\begin{array}{l}\text { Defining reciprocal value of the air gap by approximate } \\
\text { expression }\end{array}$ & difficult & impossible & easily \\
\hline Neglect of the air gap permeance between the rotor poles & difficult & possible & possible \\
\hline Disregard MMF upper harmonics and tooth kinks & difficult & difficult & easily \\
\hline Neglect of the core steel reluctance and saturation & difficult & easily & possible \\
\hline
\end{tabular}
of research of the effect assumptions onto the system performance.

TABLE I

COMPARISON OF VARIOUS MACHINE MODEL TYPES

In the table I is presented assessment possibility of allowing for and exclusion from models machine various type the assumptions.

Creation of an analytical model presupposes the obtaining of equations set describing all necessary characteristics of the machine. If one of them is added or is excluded, then is necessary to obtain new equations set. For the SRBM it is difficult, even if all assumptions are made.

FEM is a most wide-spread and simple method of the modeling of electromagnetic fields. FEM model permits to exactly compute forces of electromagnetic 
interaction. Herewith any characteristic, property of material and MMF shape isn't simplified. But exclusion from the model any attribute isn't always possible and easy.

Via programmatic model it can be enough easily done it, by changing attribute set of the model. Herewith other types of modeling are used. For example, to create the relation $R_{c}=f\left(F_{c}\right)$ it is need to use FEM, which allows to consider local saturation of iron core.

\section{REFERENCES}

[1] Y. N. Zhuravlyov, Active magnetic bearings: theory, design, applications. St. Petersburg: Polytechnica, 2003.

[2] S. Y. Loginov and Y. V. Domracheva, "Overall dimensions comparison of bearingless reluctance machine and machine with active magnetic bearings," Electrotechnical complexes and control systems, vol. 2, pp. 20-24, Feb. 2012.

[3] P. K. Hermann, "A Radial Active Magnetic Bearing Having a Rotating Drive," London Patent 1500 809, February 9, 1974.

[4] Hannian Zhang, Huangqiu Zhu, Zhibao Zhang and Zhiyi Xie, Design and Simulation of Control System for Bearingless Synchronous Reluctance Motor: Electrical Machines and Systems, 2005. ICEMS 2005. Proceedings of the Eighth International Conference, vol. 1, pp. 554-558, 2005.

[5] Zou Hualei, Diao Xiaoyan, Zhu Huangqiu, Li Tianbo and Zhu Dehong, Decoupling control of bearingless synchronous reluctance motor based on inverse system method: Robotics \& Control Systems: Control and Decision Conference (CCDC), pp. 2120-2125, 2010.

[6] A. Chiba, T. Fykao, O. Ichikawa, M. Oshima, M. Takemoto and D.G. Dorrell, Magnetic Bearings and Bearingsless Drives. ELSEVIER, 2005

[7] A. G. Slivinskaya, Electromagnets and permanent magnets. Moscow: Energy, 1972. 